\title{
On the homomorphism between the equivariant SK ring and the Burnside ring for involution
}

\author{
By Hiroaki KoshikawA \\ (Received November 28, 1984)
}

\section{Introduction.}

Let $G$ be a finite abelian group, $A(G)$ the Burnside ring and $S K_{*}^{G}$ the $G$-equivariant "cutting and pasting ring". In [3] Kosniowski proposed that we have a homomorphinsm $S K_{*}^{G} \rightarrow A(G)$ and what we can say about this homomorphism. In this note, we consider the case of $G=Z_{2}$.

Let $y=\left[Z_{2}\right] \in S K_{0}^{Z_{2}}, y_{i}=\left[R P\left(R \times \tilde{R}^{i}\right)\right] \in S K_{i}^{Z_{2}}$ for $i \geqq 0$ and $\alpha=\left[R P^{2}\right] \in$ $S K_{2}$, then we have the following relations.

THEOREM 3. For any integers $m, n \geqq 0$,
(1) $y^{2}=2 y$
(2) $y y_{2 m+1}=0$
(3) $y y_{2 m}=\alpha^{m} y$
(4) $y_{2 m}=y_{2}^{m}$
(5) $y_{2 m+1} y_{2 n}=y_{2 m+2 n+1}+\alpha^{m} y_{2 n+1}-\alpha^{m+n} y_{1}$
(6) $y_{2 m+1} y_{2 n+1}=\alpha^{m+n} y_{2}+\alpha^{m} y_{2}^{n+1}+\alpha^{n} y_{2}^{m+1}+y_{2}^{m+n+1}-2 \alpha^{m+n+1} y$, as ring structure of $S K_{*}^{Z_{2}}$.

This theorem is proved by using results of Kosniowski Theorem 1 and Corollary 2. Moreover, we have the next corollary.

COROLLARY 4. As $S K_{*}$-algebra, $S K_{*}^{Z_{2}} \cong S K_{*}\left[y, y_{1}, y_{2}, y_{3}\right] / \mathscr{I}$, where $\mathscr{I}$ is an ideal generated by the above relations with $0 \leqq m, n \leqq 1$.

Let $\phi: S K_{*}^{Z_{2}} \rightarrow A\left(Z_{2}\right)$ be a natural map $\phi([M])=[M]$. Then $\phi$ is a well-defined $S K_{*}$-algebra homomorphism. Where we regard $A\left(Z_{2}\right)$ as $S K_{*}$ -algebra induced by $\phi$.

Let $A_{1}=\alpha y_{0}-y_{0}, B_{1}=\alpha y-y, C_{1}=y_{1}-2 y_{0}+y, D_{1}=y_{2}-y_{0}$ and $E_{1}=y_{3}-y_{1}$. Then we have the following theorem.

THEOREM 10. If $\mathscr{S}$ is the $S K_{*}$-subalgebra of $S K_{*}^{Z_{2}}$ generated by $\left\{A_{1}, B_{1}\right.$, $\left.C_{1}, D_{1}, E_{1}\right\}$ then the sequence

$$
0 \rightarrow \mathscr{S} \stackrel{\iota}{\rightarrow} S K_{*}^{Z_{2}} \stackrel{\phi}{\rightarrow} A\left(Z_{2}\right) \rightarrow 0
$$

is a short exact sequence and splits as ring, where $\iota$ is an inclusion homomorphism.

This theorem is obtained by the tom Dieck's formula (Proposition 5), linear algebra and the relations of Theorem 3. We consider the structure of $S K_{*}^{Z_{2}}$ in section 2, and we shall prove the theorem 10 in section 3 . Throughout this paper $\mathrm{G}$ always denotes a finite abelian group. 


\section{The structure of $\mathrm{SK}_{*}^{\mathrm{Z}_{2}}$.}

In this section, we first recall some basic facts about the $S K_{*}^{G}$, and then we determine the $S K_{*}$-algebra structure of $S K_{*}^{Z_{2}}$.

Let $M^{n}$ be a closed $n$-dimensional smooth $G$-manifold. Let $L \subset M$ satisfy the following properties,

(1) $L$ is a $G$-invariant codimension 1 smooth submanifold of $M$,

(2) $L$ has trivial normal bundle in $M$, and

(3) the normal bundle of $L$ in $M$ is equivariantly equivalent to $L \times R$ with trivial action of $G$ on the real numbers $R$.

If we cut $M$ open along $L$, we obtain a manifold $M^{\prime}$ with boundary $\partial M^{\prime}=L+L$. Then by pasting these two copies of $L$ together via some other equivariant diffeomorphism we obtain a closed $n$-dimensional $G$-manifold $M_{1}$. We say that $M_{1}$ has been obtained from $M$ by equivariant cutting and pasting.

Definition 2.1. If $M_{1}^{n}$ has been obtained from $M^{n}$ by a finite sequence of equivariant cuttings and pastings, then we say that $M_{1}$ and $M$ are $S K^{G}$ equivalent.

This is an equivalence relation on the set of $n$-dimensional $G$-manifolds. The equivalence classes form an abelian semigroup if we use disjoint union as addition. The Grothendieck group of this semigroup is then dentoted by $S K_{n}^{G}$. If $G=\{1\}$, then $S K_{n}^{G}$ is denoted by $S K_{n}$. The equivalence class containing the $G$-manifold $M$ is denoted by $[M] . S K_{*}^{G}$ is defined as $\Sigma_{n \geq 0}$ $S K_{n}^{G}$. Then $S K_{*}^{G}$ is a module over the $S K_{*}=\Sigma_{n \geq 0} S K_{n}$, where $S K_{*}$ is the integral polynomial ring on the real projective space $\left[R P^{2}\right]$, (cf. [3]2.5.1) The module operation is given by $\left[R P^{2}\right]^{m}\left[M^{n}\right]=\left[\left(R P^{2}\right)^{m} \times M^{n}\right]$, where we consider $\left(R P^{2}\right)^{m}$ has the trivial $G$ action and $\left(R P^{2}\right)^{m} \times M^{n}$ has the diagonal $G$ action. Moreover, $S K_{*}^{G}$ is a graded ring with multiplication by $\left[M^{m}\right]$ $\left[N^{n}\right]=\left[M^{m} \times N^{n}\right]$, where $M^{m} \times N^{n}$ has also the diagonal $G$ action. The zero element of $S K_{*}^{G}$ is the class of empty set [ $\left.\phi\right]$ and the identity element is $[p t]$, where $p t$ is a point with trivial action.

For $G=Z_{2}$, the $S K_{*}$ module structure of $S K_{*}^{Z_{2}}$ has been determined by C. Kosniowski as follows.

THEOREM 1. (KOSNIOWSKI [3] 5.3.1.) $S K_{*}^{Z_{2}}$ is a free $S K_{*}$-module with basis $\left\{\left[Z_{2}\right],\left[R P\left(R \times \tilde{R}^{i}\right)\right] ; i \geqq 0\right\}$, where $\tilde{R}$ denotes the real numbers with $Z_{2}$ acting via multiplication by -1 .

COROLlARY 2. (KoSNIOWSKI [3] 5.3.7.) Let $M, M^{\prime}$ be n-dimensional $Z_{2^{-}}$ manifolds and let $F_{0}, F_{1}, \cdots, F_{n}\left(F_{0}^{\prime}, F_{1}^{\prime}, \cdots, F_{n}^{\prime}\right)$ be the fixed point sets of $M\left(M^{\prime}\right)$ of codimension $0,1, \cdots, n$ respectively. Then $M$ and $M^{\prime}$ are $S K^{Z_{2}}$ 
equivalent if and only if $\chi(M)=\chi\left(M^{\prime}\right)$ and $\chi\left(F_{i}\right)=\chi\left(F_{i}^{\prime}\right)$ for $i=0,1, \cdots$, $n$, where $\chi(M)$ is Euler characteristic of $M$.

Now, we can determine the ring structure of $S K_{*}^{Z_{2}}$ by making use of the above results. We denote $y=\left[Z_{2}\right] \in S K_{0}^{Z_{2}}, y_{i}=\left[R P\left(R \times \tilde{R}^{i}\right)\right] \in S K_{i}^{Z_{2}}$ for $i \geqq 0$ and $\alpha=\left[R P^{2}\right] \in S K_{2}$. Then we have the following relations.

THEOREM 3. For any integers $m, n \geqq 0$,

$\begin{array}{llll}\text { (1) } y^{2}=2 y & \text { (2) } y y_{2 m+1}=0 & \text { (3) } y y_{2 m}=\alpha^{m} y & \text { (4) } y_{2 m}=y_{2}^{m}\end{array}$

(5) $y_{2 m+1} y_{2 n}=y_{2 m+2 n+1}+\alpha^{m} y_{2 n+1}-\alpha^{m+n} y_{1}$

(6) $y_{2 m+1} y_{2 n+1}=\alpha^{m+n} y_{2}+\alpha^{m} y_{2}^{n+1}+\alpha^{n} y_{2}^{m+1}+y_{2}^{m+n+1}-2 \alpha^{m+n+1} y$, as ring structure of $S K_{*}^{Z_{2}}$.

Proof. Compare the Euler characteristics of the fixed point sets of both sides of these equalities. Then we can obtain the above relations by Corollary 2.

q. e. d.

Next we consider the $S K_{*}$-algebra structure of $S K_{*}^{Z_{2}}$. Then we can reduce the relation (5) to the following.

(5') $y_{2 m+3}=y_{3} y_{2}^{m}-\left(y_{3}-\alpha y_{1}\right) \sum_{i=1}^{m} \alpha^{i} y_{2}^{m-i}$ for $m \geqq 1$.

This is proved by induction on $\mathrm{m}$. Therefore any element of $S K_{*}^{Z_{2}}$ can be expressed as a $S K_{*}$-polynomial of $y, y_{1}, y_{2}, y_{3}$ with relations of Theorem 3 . And $y, y_{1}, y_{2}, y_{3}$ have no any other relations, because Euler characteristics of fixed point sets are $S K^{Z_{2}}$ invariant. So we have next corollary.

COROLLARY 4. As $S K_{*}$-algebra $S K_{*}^{Z_{2}} \cong S K_{*}\left[y, y_{1}, y_{2}, y_{3}\right] / \mathscr{I}$, where $\mathscr{I}$ is an ideal generated by the relations with $0 \leqq m, n \leqq 1$ of Theorem 3 .

\section{The relations between $S K_{*}^{Z_{2}}$ and $A\left(Z_{2}\right)$.}

Let $M$ and $N$ be the closed smooth $G$-manifolds. We define another equivalence relation as follows.

$M \sim N$ if and only if the $H$-fixed point sets $M^{H}$ and $N^{H}$ for all subgroups $H$ of $G$ have the same Euler characteristics $\chi\left(M^{H}\right)$ and $\chi\left(N^{H}\right)$. Denote by $A(G)$ the set of equivalence classes under this equivalence relation, and denote by $[M] \in A(G)$ the class of $M$ (we use conveniently same notation as the element of $\left.S K_{*}^{G}\right)$. The disjoint union and the cartesian product of $G$-manifolds induce an addition and multiplication on $A(G)$. Then $A(G)$ becomes a commutative ring with identity $[p t]$.

Definition 3.1. We call $A(G)$ the Burnside ring of $G$.

Let $M$ be a $G$ manifold and $H$ be a subgroup of $G$. Then we define $M_{H}=\left\{x \in M \mid G_{x}=H\right\}$, where $G_{x}$ denotes the isotropy group at $x$. Now we note that we consider only $G$ a finite abelian group. So the next formula is 
the special case of tom Dieck's one ([2], 5.5.1)

Proposition 5. Additively, $A(G)$ is the free abelian group on $[G / H]$ and any element $[M] \in A(G)$ have the relation

$$
[M]=\sum_{H \subset G} \chi\left(M_{H} / G\right)[G / H]
$$

By this formula, we have the following.

Lemma 6. $\quad A\left(Z_{p}\right) \cong Z[x] /\left(x^{2}-p x\right)$ for any prime integer $p$.

Proof. $A\left(Z_{p}\right)$ is a free abelian group generated by $\left[Z_{p}\right]$ and $\left[Z_{p} / Z_{p}\right]$. We set $x=\left[Z_{p}\right], 1=\left[Z_{p} / Z_{p}\right]$. Then $x^{2}=\left[Z_{p} \times Z_{p}\right]=\chi\left(Z_{p}\right)\left[Z_{p}\right]=p x$, because the action of $Z_{p}$ to $Z_{p} \times Z_{p}$ is the diagonal. q. e.d.

Definition 3.2. Let $[M] \in S K_{*}^{Z_{2}}$, then $[M]$ can be naturally regarded as the element of $A\left(Z_{2}\right)$. We denote this correspondence by $\phi: S K_{*}^{Z_{2}} \rightarrow A$ $\left(Z_{2}\right)$. Then $\phi$ is a well-defined ring homomorphism by Corollary 2 .

By this ring homomorphism, the generators of $S K_{*}^{Z_{2}}$ are mapped as follows.

Lemma 7. $\phi(y)=x, \phi\left(y_{2 n+1}\right)=2-x$, and $\phi\left(y_{2 n}\right)=1$ for $n \geqq 0$, where $x=\left[Z_{2}\right]$ and $1=\left[Z_{2} / Z_{2}\right]$.

Proof. $\phi(y)=x$ is a trivial. Next we recall $y_{2 n+1}=\left[R P\left(R \times \tilde{R}^{2 n+1}\right)\right]$. Let $\phi\left(y_{2 n+1}\right)=a+b x$ for $a, b \in Z$. Then $\chi\left(R P\left(R \times \tilde{R}^{2 n+1}\right)\right)=0$ and $\chi(R P$ $\left.\left(R \times \tilde{R}^{2 n+1}\right)^{Z_{2}}\right)=2$, so $a=2$ and $b=-1$. Therefore $\phi\left(y_{2 n+1}\right)=2-x$. Similarly we obtain $\phi\left(y_{2 n}\right)=1$.

q. e. d.

Next let us calculate Ker $\phi$.

LEMMA 8. $\quad K e r \phi$ is generated by $\left\{\alpha^{i} y_{2 j}-y_{0}, \alpha^{k} y_{2 l+1}-2 y_{0}+y, \alpha^{m} y-y\right\}$, where $i, j, k, l \geqq 0$ (except for $i=j=0$ ) and $m \geqq 1$.

Proof. For any fixed $n \geqq 0$, let $[M]$ be in $\operatorname{Ker} \phi$ and let it be the $S K_{*}$ linear combination as follows,

$[M]=\sum_{0 \leqq i+j \leqq n} a_{i}^{j} \alpha^{i} y_{j}+\sum_{0 \leqq k+l \leqq n} b_{k}^{l} \alpha^{k} y_{2 l+1}+\sum_{0 \leqq m \leqq n} c_{m} \alpha^{m} y$, for $a_{i}^{j}, b_{k}^{l}, \quad c_{m} \in Z$.

Now $\phi(\alpha)=1$, so by Lemma 7 ,

$\phi([M])=\sum_{0 \leqq i+j \leqq n} a_{i}^{j}+\sum_{0 \leqq k+l \leqq n} b_{k}^{l}+\left(\sum_{0 \leqq m \leqq n} c_{m}-\sum_{0 \leqq k+l \leqq n} b_{k}^{l}\right) x$.

Then we have the conclusions by the linearly independent solutions of next simultaneous equations.

$$
\left\{\begin{array}{l}
\sum_{0 \leqq i+j \leqq n} a_{i}^{j}+\underset{0 \leqq k+l \leqq n}{2 \sum_{k} b_{k}^{l}=0} \\
\sum_{0 \leqq m \leqq n} \mathrm{c}_{m}-\sum_{0 \leqq k+l \leqq n} b_{k}^{l}=0 .
\end{array}\right.
$$

Since $S K_{*} \subset S K_{*^{2}}^{Z_{2}}$, we may consider $A\left(Z_{2}\right)$ as $S K_{*}$-algebra via $\phi$ (cf. 
[1] Chapter 2). In this case, for $[M] \in S K_{*}, \quad[N] \in A\left(Z_{2}\right)[M][N]=$ $\phi([M])[N]=[M \times N]$ and $\phi$ is algebra homomorphism.

Now we reduce the above generators in order to get the minimal set of generators of $\mathrm{Ker} \phi$ as $S K_{*}$-subalgebra.

Let $A_{i}=\alpha^{i} y_{0}-y_{0}, B_{j}=\alpha^{j} y-y, C_{1}=y_{1}-2 y_{0}+y, D_{k}=y_{2 k}-y_{0}$ and $E_{l}=$ $y_{2 l+1}-y_{1}$ for $i, j, k, l \geqq 1$. Then we can reduce these relations as follows.

LEMMA 9.

$$
\begin{gathered}
A_{i}=\sum_{s=1}^{i} \alpha^{i-s} A_{1} \\
\mathrm{~B}_{j}=\sum_{s=1}^{j} \alpha^{j-s} B_{1} \\
D_{k}=\sum_{s=0}^{k-1}\left(\begin{array}{c}
k \\
s
\end{array}\right) D_{1}^{k-s} \\
E_{l+1}=E_{1}\left(D_{1}+1\right)^{l}+\sum_{s=1}^{l}\left(D_{1}+1\right)^{l-s}\left\{E_{1}\left(2-\alpha^{s}\right)+\left(\alpha^{s}-1\right)\left(2 A_{1}-B_{1}+\right.\right.
\end{gathered}
$$
$\left.\left.\alpha C_{1}-C_{1}\right)\right\}$, where $i, j, k, l \geqq 1$.

Proof. We can easily obtain (3.1) and (3.2) by induction on $i$ and $j$ respectively. We have (3.3) by the relation

$$
D_{k+1}=D_{k} D_{1}+D_{k}+D_{1} \text {. }
$$

In order to get (3.4), we deform $E_{l+1}$ as follows.

$$
\begin{aligned}
E_{l+1} & =y_{2 l+3}-y_{1} \\
& =y_{2 l+1} y_{2}-\alpha^{l} y_{3}+\alpha^{l+1} y_{1}-y_{1} \\
& =\left(y_{2 l+1}-y_{1}\right)\left(y_{2}-y_{0}\right)+\left(y_{2 l+1}-y_{1}\right)+y_{1} y_{2}-\alpha^{l}\left(y_{3}-\alpha y_{1}\right)-y_{1} \\
& =E_{l} D_{1}+E_{l}+2 y_{3}-\alpha y_{1}-\alpha^{l}\left(y_{3}-\alpha y_{1}\right)-y_{1} \\
& =E_{l} D_{1}+E_{l}+2 E_{1}-\alpha^{l} E_{1}+\left(\alpha^{l}-1\right)\left(\alpha y_{1}-y_{1}\right),
\end{aligned}
$$

where $\quad \alpha y_{1}-y_{1}=2\left(\alpha y_{0}-y_{0}\right)-(\alpha y-y)+\alpha\left(y_{1}-2 y_{0}+y\right)-\left(y_{1}-2 y_{0}+y\right)=2 A_{1}-$ $B_{1}+\alpha C_{1}-C_{1}$. We set $\beta=D_{1}+1, \gamma_{l}=E_{1}\left(2-\alpha^{l}\right)+\left(\alpha^{l}-1\right)\left(2 A_{1}-B_{1}+\alpha C_{1}-\right.$ $C_{1}$ ), then $E_{l+1}=E_{l} \beta+\gamma_{l}$. Thus we can obtain (3.4) by induction on $l$.

q. e.d.

While we have

(3.5) $\alpha^{i} y_{2 j}-y_{0}=\alpha^{i} D_{j}+A_{i}$, and

(3.6) $\alpha^{k} y_{2 l+1}-2 y_{0}+y=\alpha^{k} E_{l}+2 A_{k}-B_{k}+\alpha^{k} C_{1}$.

Therefore, by Lemma 9, we see that $A_{1}, B_{1}, C_{1}, D_{1}$ and $E_{1}$ are minimal set of generators of $\mathrm{Ker} \phi$ as $S K_{*}$-subalgebra of $S K_{*}^{Z_{2}}$. Then we have the following theorem.

THEOREM 10. If $\mathscr{S}$ is the $S K_{*}$-subalgebra of $S K_{*}^{Z_{2}}$ generated by $\left\{A_{1}\right.$, $\left.B_{1}, C_{1}, D_{1}, E_{1}\right\}$ then the sequence

$$
0 \rightarrow \mathscr{\iota} \stackrel{\iota}{\rightarrow} S K_{*}^{Z_{2}} \stackrel{\phi}{\rightarrow} A\left(Z_{2}\right) \rightarrow 0
$$

is a short exact sequence and splits as ring, where $\iota$ is an inclusion homo- 


\section{morphism.}

Proof. By the above argument $\mathscr{S}=\operatorname{Ker} \phi$, so the exactness is trivial. The split map $\psi: A\left(Z_{2}\right) \rightarrow S K_{*}^{Z_{2}}$ is given by $\psi(1)=y_{0}$, and $\psi(x)=y$. By Theorem 3 and Lemma 7 , we see that $\psi$ is a split ring homomorphism.

q.e.d.

\section{References}

[1] M. F. ATIYAH, and I. G. MAC DONALD: Introduction to commutative algebra, Addison-Wesley, 1969.

[ 2 ] T. TOM DIECK : Transformation groups and representation theory, Springer Lec. Notes 766, 1979.

[ 3 ] C. Kosniowski : Actions of finite abelian groups, Pitman, 1978.

Department of Mathematics

Faculty of Education

Chiba University 